\title{
Stability analysis and observer design for one-sided Lipschitz descriptor systems with time-varying delay
}

\author{
Jing Hao, Yali Dong \\ School of Science, Tianjin Polytechnic University, Tianjin, 300387, China \\ E-mail:dongyl@vip.sina.com
}

\begin{abstract}
This paper investigates the problem of stability analysis and observer design for nonlinear descriptor systems with time-varying delay. In the systems, the nonlinear function satisfies the one-sided Lipschitz condition and the quadratic internal boundary condition. The disturbance is considered in both the state and the output equation. Using one-sided Lipschitz condition, the quadratic internal boundary condition, and the generalized Lyapunov method, we establish the non-strict bilinear matrix inequality (BMI)-based condition. We change the condition into strict bilinear matrix inequality (BMI) condition. Furthermore, we give the linear matrix inequality-based condition to ensure the gradual convergence of state estimation error and to accomplish robustness against $L_{2}$ norm bounded disturbances by utilizing change of variables for straight forward computation of the observer gain matrix. Finally, a numerical example is given to verify the effectiveness of the observer design scheme.
\end{abstract}

Keywords: Descriptor systems; observer design; asymptotically stability; one-sided Lipschitz condition; timevarying delay.

\section{Introduction}

Descriptor systems, also known as singular systems, implicit systems or generalized state space systems, are widely used in many fields. Such as power systems, network and circuit analysis systems, mechanical systems, economic systems, etc. Compared to the traditional state space model, descriptor systems can describe a broader range of system types much more accurately. Therefore, the basic state and theory of singular systems are researched in a large number of studies, such as stability and control problems [1-4]. In [1], admissibility analysis and control synthesis for descriptor systems with random abrupt changes were given. In [2], the reliable passive control for singular systems with time-varying delays was considered. In [3], robust observer-based output feedback control for fuzzy descriptor systems was studied.

Since last decade, many research have focused on observer design for nonlinear dynamical systems, inspired by the fact that state estimation can be used for control, diagnosis and supervision purposes. The conventional Lipschitz condition is commonly used in the existing nonlinear observer design. However, a major limitation in the existing results for Lipschitz nonlinear systems is that most of them work only for adequately small values of the Lipschitz constant [5]. In order to overcome the limitation, the so-called one-sided Lipschitz condition was first introduced in [6] for nonlinear state estimation. In recent years, one-sided Lipschitz nonlinear systems have become an important class of nonlinear systems because compared to the Lipschitz systems, one-sided Lipschitz nonlinear systems represent a more general form. Several works on state observers for this type of systems were reported in [5-11]. In [7], Liu et al. investigated state observer design problem for a class of nonlinear dynamical systems with interval time-varying delay that satisfies the one-sided Lipschitz condition. In [8], full-order and reduced-order observers for one-sided Lipschitz nonlinear systems were given through the use of Riccati equations. In [10], nonlinear $H_{\infty}$ observer design for one-sided Lipschitz systems was involved.

On the other hand, time-delay is a common phenomenon encountered in various practical systems, such as chemical engineering systems, distributed networks, and neural networks. It has been regarded as a main source of instability and poor performance. Therefore, much attention has been paid to the problem of observer design for time-delay systems. Many research results have been reported in the literature [12-15]. However, none of these observer design schemes can be applied to the one-sided Lipschitz descriptor systems with time-varying delay.

In this paper, a novel observer design method is proposed for the one-sided Lipschitz descriptor system with time delay. A reliable state estimation can be ensured in both state and output equations. Using the one-sided Lipschitz condition, the quadratic internal boundary conditions, and the generalized Lyapunov method, first, we propose a non-strict bilinear matrix inequality (BMI)-based condition, which is further transformed into strict 
bilinear matrix inequality (BMI)-based condition using a proper change of variables. Furthermore, we develop the linear matrix inequality (LMI)-based condition to ensure asymptotic convergence of the state estimation error to origin and to accomplish robustness against $L_{2}$ norm bounded disturbances. Finally, a simulation study is conducted to verify the effectiveness of the proposed method.

The main structure of the paper is arranged as follows: In Section 2, the descriptor system is described and the observer design is carried out, and the assumptions and lemmas needed for the theorem are given. In Section 3, the observer design scheme for the one-sided Lipschitz descriptor system is presented with strict and nonstrict BMI conditions and LMI conditions. In Section 4, we show the effectiveness of the proposed observer design method through an illustrative example.

Notations: The notations used in this paper are standard. The superscript ' $T$ ' denotes matrix transpose, $R^{n}$ denotes the $n$-dimensional real Euclidean space. The notation $P>0(P<0)$ means that the matrix is positive definite (negative definite). The notation ' $*$ ' always denotes the symmetric block in one symmetric matrix. $\|\cdot\|$ represent the Euclidean norm, and $\|\cdot\|_{2}=\sqrt{\int_{0}^{\infty}\|\cdot\|^{2} d t}$.

\section{System description}

Consider a nonlinear descriptor system as follows:

$$
\begin{gathered}
E \dot{x}(t)=A_{1} x(t)+A_{2} x(t-d(t))+\varphi(t, x)+B \omega(t), \\
y=C x(t)+D \omega(t),
\end{gathered}
$$

where $x(t) \in R^{n}, y \in R^{p}$ and $\omega(t) \in R^{q}$ represent the state, the output and the disturbance vectors for the above system. $d(t)$ is a time-varying continuous function that satisfies

$$
0 \leq d(t) \leq d, \quad \dot{d}(t) \leq \mu .
$$

The nonlinear function $\varphi(t, x) \in R^{n}$ denotes the nonlinearities in the state. The matrices $A_{1} \in R^{n \times n}, A_{2} \in R^{n \times n}, B \in R^{n \times q}, C \in R^{p \times n}, D \in R^{p \times q}$ have constant entries, $E \in R^{n \times n}$ is singular with $\operatorname{rank}(E)=r<n$.

Assumption 1.

(a) The nonlinear function $\varphi(t, x)$ satisfies the one-sided Lipschitz condition, given by:

$$
\left\langle\varphi\left(t, x_{1}\right)-\varphi\left(t, x_{2}\right), x_{1}-x_{2}\right\rangle \leq \rho\left\|x_{1}-x_{2}\right\|^{2},
$$

for a real scalar $\rho$ and $x_{1}, x_{2} \in R^{n}$, where $\rho$ is the one-sided Lipschitz constant which can be positive or negative.

(b) The nonlinear function $\varphi(t, x)$ satisfies the quadratic inner boundedness, given by:

$$
\left(\varphi\left(t, x_{1}\right)-\varphi\left(t, x_{2}\right)\right)^{T}\left(\varphi\left(t, x_{1}\right)-\varphi\left(t, x_{2}\right)\right) \leq \delta_{1}\left\|x_{1}-x_{2}\right\|^{2}+\delta_{2}\left\langle x_{1}-x_{2}, \varphi\left(t, x_{1}\right)-\varphi\left(t, x_{2}\right)\right\rangle,
$$

where $\delta_{1}$ and $\delta_{2}$ are scalars.

Assumption 2. The disturbance $\omega(t)$ has bounded $L_{2}$ norm.

Now, consider a state observer described by:

$$
\begin{gathered}
E \dot{\hat{x}}(t)=A_{1} \hat{x}(t)+A_{2} \hat{x}(t-d(t))+\varphi(t, \hat{x})+L(y-\hat{y}), \\
\hat{y}=C \hat{x}(t),
\end{gathered}
$$

where $\hat{x}(t)$ is the estimated state, $\hat{y}$ is output vectors for the observer. $L \in R^{n \times p}$ represents the observer gain matrix to be designed. Define the state estimated error

$$
e(t)=x(t)-\hat{x}(t)
$$

Then the error system can be written in the form as follows:

$$
E \dot{e}(t)=\left(A_{1}-L C\right) e(t)+A_{2} e(t-d(t))+\Phi(t, x, \hat{x})+(B-L D) \omega(t),
$$

where $\Phi(t, x, \hat{x})=\varphi(t, x)-\varphi(t, \hat{x})$.

Lemma 1. [16] A matrix $A \in R^{n \times n}$ is invertible if there is a matrix norm $\|\cdot\|$ such that $\|I-A\|<1$.

The symbol $\|\cdot\|$ in the above lemma represents any matrix norm.

\section{Main results}


In this section, our objective is to establish less conservative criteria for the asymptotically stability of the system (7).

Theorem 1. Consider a one-sided Lipschitz time-delay nonlinear system of the form (1) and (2) satisfying Assumptions 1 and 2. Suppose there exist scalars $\gamma>0, \varepsilon_{1}>0, \varepsilon_{2}>0$, and matrices $P \in R^{n \times n}, L \in R^{n \times p}$, positive definite matrix $Q \in R^{n \times n}, R \in R^{n \times n}, S \in R^{n \times n}$, such that the following inequalities are satisfied under $\operatorname{rank}\left(P^{T} E\right)=\operatorname{rank}(E)$ :

$$
P^{T} E=E^{T} P \geq 0,
$$

$$
\Omega=\left[\begin{array}{ccccccc}
\Omega_{11} & P^{T} A_{2} & 0 & \Omega_{14} & 0 & P^{T}(B-L D) & I \\
* & -(1-\mu) Q & 0 & 0 & 0 & 0 & 0 \\
* & * & -R & 0 & 0 & 0 & 0 \\
* & * & * & -\varepsilon_{2} I & 0 & 0 & 0 \\
* & * & * & * & -S & 0 & 0 \\
* & * & * & * & * & -\gamma I & 0 \\
* & * & * & * & * & * & -\gamma I
\end{array}\right]<0,
$$

where

$$
\begin{gathered}
\Omega_{11}=\left(A_{1}-L C\right)^{T} P+P^{T}\left(A_{1}-L C\right)+Q+R+d^{2} S+\varepsilon_{1} \rho I+\varepsilon_{2} \delta_{1} I, \\
\Omega_{14}=P^{T}-\frac{1}{2} \varepsilon_{1} I-\frac{1}{2} \varepsilon_{2} \delta_{2} I .
\end{gathered}
$$

Then, the error dynamics (7) is asymptotically stable if $\omega(t)=0$. Moreover, the $L_{2}$ gain from the disturbance $\omega(t)$ to the state estimation error $e=x-\hat{x}$ is less than $\gamma$ for all time.

Proof: Choose the following Lyapunov-Krasovskii functional

$$
V(t, e)=e^{T}(t) E^{T} P e(t)+\int_{t-d(t)}^{t} e^{T}(s) Q e(s) d s+\int_{t-d}^{t} e^{T}(s) \operatorname{Re}(s) d s+d \int_{-d}^{0} \int_{t+\theta}^{t} e^{T}(s) \operatorname{Se}(s) d s d \theta,
$$

where $P^{T} E=E^{T} P \geq 0, \operatorname{rank}\left(P^{T} E\right)=\operatorname{rank}(E)$. Note that $V(t, e)=0$, if and only if $e(t)=0$, and $V(t, e)>0$, otherwise. Taking the time derivative of $V(t, e)$ along the trajectory of the system (7) yields:

$$
\begin{aligned}
\dot{V}(t, e)= & \dot{e}^{T}(t) E^{T} P e(t)+e^{T}(t) P^{T} E \dot{e}(t)+e^{T}(t) Q e(t)-(1-\mu) e^{T}(t-d(t)) Q e(t-d(t)) \\
& +e^{T}(t) \operatorname{Re}(t)-e^{T}(t-d) \operatorname{Re}(t-d)+d^{2} e^{T}(t) \operatorname{Se}(t)-d \int_{t-d}^{t} e^{T}(s) \operatorname{Se}(s) d s .
\end{aligned}
$$

Let

$$
J=\dot{V}+\gamma^{-1} e^{T} e-\gamma \omega^{T} \omega .
$$

Substituting (11) into (12), it can be obtained that

$$
J \leq \xi^{T}(t) \Omega_{1} \xi(t)
$$

where

$$
\begin{gathered}
\xi^{T}(t)=\left[\begin{array}{cccccc}
e^{T}(t) & e^{T}(t-d(t)) & e^{T}(t-d) & \Phi^{T}(t, x, \hat{x}) & {\left[\int_{t-d}^{t} e(s) d s\right]^{T}} & \omega^{T}(t)
\end{array}\right], \\
\Omega_{1}=\left[\begin{array}{cccccc}
\Omega_{11} & P^{T} A_{2} & 0 & P^{T} & 0 & P^{T}(B-L D) \\
* & -(1-\mu) Q & 0 & 0 & 0 & 0 \\
* & * & -R & 0 & 0 & 0 \\
* & * & * & 0 & 0 & 0 \\
* & * & * & * & -S & 0 \\
* & * & * & * & * & -\gamma I
\end{array}\right], \\
\Omega_{11}=\left(A_{1}-L C\right)^{T} P+P^{T}\left(A_{1}-L C\right)+Q+R+d^{2} S+\gamma^{-1} I .
\end{gathered}
$$

According to Assumption 1 , for any $\varepsilon_{1}>0$ the following inequality holds:

$$
\varepsilon_{1} \rho e^{T}(t) e(t)-\varepsilon_{1} e^{T}(t) \Phi(t, x, \hat{x}) \geq 0,
$$

which further can be written as

$$
\xi^{T}(t) \Omega_{2} \xi(t) \geq 0
$$

where 
Assumption 1(b) reveals that

$$
\Omega_{2}=\left[\begin{array}{cccccc}
\varepsilon_{1} \rho I & 0 & 0 & -\frac{1}{2} \varepsilon_{1} I & 0 & 0 \\
* & 0 & 0 & 0 & 0 & 0 \\
* & * & 0 & 0 & 0 & 0 \\
* & * & * & 0 & 0 & 0 \\
* & * & * & * & 0 & 0 \\
* & * & * & * & * & 0
\end{array}\right] .
$$

$$
\varepsilon_{2}\left[\delta_{1} e^{T}(t) e(t)-\delta_{2} e^{T}(t) \Phi(t, x, \hat{x})-\Phi^{T}(t, x, \hat{x}) \Phi(t, x, \hat{x})\right] \geq 0, \quad \varepsilon_{2}>0,
$$

which further can be written as

$$
\xi^{T}(t) \Omega_{3} \xi(t) \geq 0
$$

where

$$
\Omega_{3}=\left[\begin{array}{cccccc}
\varepsilon_{2} \delta_{1} I & 0 & 0 & -\frac{1}{2} \varepsilon_{2} \delta_{2} I & 0 & 0 \\
* & 0 & 0 & 0 & 0 & 0 \\
* & * & 0 & 0 & 0 & 0 \\
* & * & * & -\varepsilon_{2} I & 0 & 0 \\
* & * & * & * & 0 & 0 \\
* & * & * & * & * & 0
\end{array}\right] .
$$

Combining the matrix inequalities (13)-(15), we can obtain that

$$
J \leq \xi^{T}(t) \bar{\Omega} \xi(t),
$$

where

$$
\begin{gathered}
\bar{\Omega}=\left[\begin{array}{cccccc}
\bar{\Omega}_{11} & P^{T} A_{2} & 0 & P^{T}-\frac{1}{2} \varepsilon_{1} I-\frac{1}{2} \varepsilon_{2} \delta_{2} I & 0 & P^{T}(B-L D) \\
* & -(1-\mu) Q & 0 & 0 & 0 & 0 \\
* & * & -R & 0 & 0 & 0 \\
* & * & * & -\varepsilon_{2} I & 0 & 0 \\
* & * & * & * & -S & 0 \\
* & * & * & * & * & -\gamma I
\end{array}\right], \\
\bar{\Omega}_{11}=\left(A_{1}-L C\right)^{T} P+P^{T}\left(A_{1}-L C\right)+Q+R+d^{2} S+\gamma^{-1} I+\varepsilon_{1} \rho I+\varepsilon_{2} \delta_{1} I .
\end{gathered}
$$

By applying Schur's complement and (9), we have that

$$
\bar{\Omega}<0 .
$$

So it follows that

$$
J \leq \xi^{T}(t) \Omega_{1} \xi(t)<0 .
$$

Under $\omega(t)=0, \dot{V}(t, e)<0$ is implied, that is to say, the error converges to origin asymptotically. Integrating (13) from 0 to $\infty$, it is implicit to conclude that

$$
\frac{\|e(t)\|_{2}}{\|\omega(t)\|_{2}}<\gamma .
$$

Explicitly, the $L_{2}$ gain from the disturbance $\omega(t)$ to the state estimation error $e(t)$ is less than $\gamma$, which completes the proof of Theorem 1 .

Theorem 2. Consider a one-sided Lipschitz time-delay nonlinear system of the form (1) and (2) satisfying Assumptions 1 and 2. Suppose there exist scalars $\gamma>0, \varepsilon_{1}>0, \varepsilon_{2}>0$, symmetric matrix $X \in R^{n \times n}$, $L \in R^{n \times p}, Y \in R^{n \times n}$, positive definite matrix $Q \in R^{n \times n}, R \in R^{n \times p}, S \in R^{n \times n}$, such that the following inequalities hold: $X>0$, 


$$
\Sigma=\left[\begin{array}{ccccccc}
\Sigma_{11} & E^{T} X A_{2}+Y^{T} E_{\perp} A_{2} & 0 & \Sigma_{14} & 0 & \Sigma_{16} & I \\
* & -(1-\mu) Q & 0 & 0 & 0 & 0 & 0 \\
* & * & -R & 0 & 0 & 0 & 0 \\
* & * & * & -\varepsilon_{2} I & 0 & 0 & 0 \\
* & * & * & * & -S & 0 & 0 \\
* & * & * & * & * & -\gamma I & 0 \\
* & * & * & * & * & * & -\gamma I
\end{array}\right]<0,
$$

where $E_{\perp}$ is the orthogonal complement of $E$ and

$$
\begin{aligned}
\Sigma_{11}= & A_{1}^{T} X E+E^{T} X A_{1}+A_{1}^{T} E_{\perp} Y+Y^{T} E_{\perp} A_{1}^{T}-C^{T} L^{T} X E-E^{T} X L C-C^{T} L^{T} E_{\perp}^{T} Y-Y^{T} E_{\perp} L C \\
& +Q+R+d^{2} S+\varepsilon_{1} \rho I+\varepsilon_{2} \delta_{1} I, \\
\Sigma_{14}= & X E+E_{\perp}^{T} Y-\frac{1}{2} \varepsilon_{1} I-\frac{1}{2} \varepsilon_{2} \delta_{2} I, \\
\Sigma_{16}= & E^{T} X B+Y^{T} E_{\perp} B-Y^{T} E_{\perp} L D-E^{T} X L D .
\end{aligned}
$$

Then, the error dynamics (7) is asymptotically stable, if $\omega(t)=0$. Moreover, the $L_{2}$ gain from the disturbance $\omega(t)$ to the state estimation error $e=x-\hat{x}$ is less than $\gamma$ for all time.

Proof: Applying the transformation $P=X E+E_{\perp}^{T} Y$ to (9) implies (19). Note that

$$
V(t, e)=e^{T}(t) E^{T} X E e(t)+\int_{t-d(t)}^{t} e^{T}(s) Q e(s) d s+\int_{t-d}^{t} e^{T}(s) \operatorname{Re}(s) d s+d \int_{-d}^{0} \int_{t+\theta}^{t} e^{T}(s) \operatorname{Se}(s) d s d \theta \geq 0,
$$

because $E^{T} P=E^{T} X E$ for $X>0$ and $Y$. The remaining part of the proof is same as for the proof of Theorem 1 . Theorem 3. Consider a one-sided Lipschitz nonlinear system of the form (1) and (2) satisfying Assumptions 1 and 2. Suppose there exist scalars $\gamma>0, \varepsilon_{1}>0, \varepsilon_{2}>0$, symmetric matrix $0<X \in R^{n \times n}, L \in R^{n \times p}, Y \in R^{n \times n}$, positive definite matrix $G \in R^{n \times p}, Q \in R^{n \times n}, R \in R^{n \times n}, S \in R^{n \times n}$, such that the inequalities hold:

$$
\Xi=\left[\begin{array}{ccccccc}
I & I-E^{T} X-Y E_{\perp} \\
* & I &
\end{array}\right]>0,
$$

where $E_{\perp}$ is the orthogonal complement of $E$ and

$$
\begin{aligned}
& \Xi_{11}=A_{1}^{T} X E+E^{T} X A_{1}+A_{1}^{T} E_{\perp}^{T} Y+Y^{T} E_{\perp} A_{1}^{T}-C^{T} G^{T}-G C+Q+R+d^{2} S+\varepsilon_{1} \rho I+\varepsilon_{2} \delta_{1} I, \\
& \Xi_{14}=X E+E_{\perp}^{T} Y-\frac{1}{2} \varepsilon_{1} I-\frac{1}{2} \varepsilon_{2} \delta_{2} I, \\
& \Xi_{16}=E^{T} X B+Y^{T} E_{\perp} B-G D .
\end{aligned}
$$

The nonlinear observer (5) and (6) with observer gain matrix $L=\left(E^{T} X+Y E_{\perp}\right)^{-1} G$ ensures

(a) asymptotic convergence of $\hat{x}$ to $x$, if $\omega(t)=0$;

(b) the $L_{2}$ gain from the disturbance $\omega(t)$ to the state estimation error $e=x-\hat{x}$ less than $\gamma$ for all time.

Proof: Applying the transformation $G=P^{T} L$ to (9) reveal 
where

$$
\tilde{\Omega}=\left[\begin{array}{ccccccc}
\Omega_{11} & P^{T} A_{2} & 0 & P^{T}-\frac{1}{2} \varepsilon_{1} I-\frac{1}{2} \varepsilon_{2} \delta_{2} I & 0 & P^{T} B-G D & I \\
* & -(1-\mu) Q & 0 & 0 & 0 & 0 & 0 \\
* & * & -R & 0 & 0 & 0 & 0 \\
* & * & * & -\varepsilon_{2} I & 0 & 0 & 0 \\
* & * & * & * & -S & 0 & 0 \\
* & * & * & * & * & -\gamma I & 0 \\
* & * & * & * & * & * & -\gamma I
\end{array}\right]<0,
$$

To ensure the matrix $P$ invertible, we require that the following inequality holds:

$$
\left[\begin{array}{cc}
I & I-P^{T} \\
* & I
\end{array}\right]>0 .
$$

Since we are using the spectral matrix norm (matrix 2-norm) through out this paper, based on Lemma 1, a sufficient condition for nonsingularity of $P$ is that $\|I-P\|=\sigma_{\max }(I-P)<1$. This is equivalent to $I-(I-P)^{T}(I-P)>0$. Thus, using Schur's complement, LMI (23) guarantees the nonsingularity of $P$. Still using the transformation $P=X E+E_{\perp}^{T} Y$ to (22) and (23), (21) and (20) can be get. The observer gain matrix can be computed by solving $L=\left(E^{T} X+Y E_{\perp}\right)^{-1} G$. The remainder of the proof is same as for the proof of Theorem 2 .

\section{Numerical example}

A simulation example is provided in this section to illustrate advantages of our schemes and to demonstrate effectiveness of the proposed observer synthesis approach for the descriptor one-sided Lipschitz systems.

Consider a system of the form (1) and (2) with the following parameters:

$$
\begin{gathered}
E=\left[\begin{array}{ll}
1 & 0 \\
0 & 0
\end{array}\right], A_{1}=\left[\begin{array}{cc}
1 & 12 \\
-6 & -15
\end{array}\right], A_{2}=\left[\begin{array}{cc}
1 & 0 \\
0 & -1
\end{array}\right], B=\left[\begin{array}{lll}
1 & 0 & 0 \\
0 & 1 & 0
\end{array}\right], \\
C=\left[\begin{array}{ll}
2 & 0
\end{array}\right], D=\left[\begin{array}{lll}
0 & 0 & 1
\end{array}\right], \varphi(t, x)=\left[\begin{array}{c}
0.05 x_{1}^{2} \\
0
\end{array}\right] .
\end{gathered}
$$

It can be verified that $\varphi(t, x)$ satisfies (3) for $\rho_{1}=0.225$, because

$$
(x-\hat{x})^{T}(\varphi(t, x)-\varphi(t, \hat{x}))=0.05\left(x_{1}+\hat{x}_{1}\right)\left(x_{1}-\hat{x}_{1}\right)^{2} \leq 0.1 \sqrt{5}\left(x_{1}-\hat{x}_{1}\right)^{2},
$$

is valid for the region $\|x\| \leq 5$. Furthermore, $\varphi(t, x)$ satisfies (4) for $\delta_{1}=0.25$ and $\delta_{2}=0$ for $\|x\| \leq 5$. By using Robust Control Toolbox of MATLAB, we can get the solution of the linear matrix inequality in Theorem 3 as follows:

$$
\begin{gathered}
X=\left[\begin{array}{cc}
1.0573 & -0.2554 \\
-0.2554 & 15.5737
\end{array}\right], Y=\left[\begin{array}{ll}
1.2587 & 0.0408 \\
0.0408 & 0.4979
\end{array}\right], \quad G=\left[\begin{array}{c}
12.9066 \\
-3.5032
\end{array}\right], \\
Q=\left[\begin{array}{cc}
9.2806 & 3.3544 \\
3.3544 & 10.1149
\end{array}\right], R=\left[\begin{array}{ll}
7.6708 & 2.5228 \\
2.5228 & 8.1795
\end{array}\right], S=\left[\begin{array}{cc}
22.8644 & 1.3416 \\
1.3416 & 19.8425
\end{array}\right], \\
\gamma=20.9487, \quad \varepsilon_{1}=5.1511, \quad \varepsilon_{2}=10.8105, \quad L=\left[\begin{array}{l}
10.3752 \\
-8.7056
\end{array}\right] .
\end{gathered}
$$

Figure 1 plots states of the plant and the observer against time for initial conditions $x(0)=\left[\begin{array}{ll}2 & 1.2\end{array}\right]^{T}$ and $\hat{x}(0)=\left[\begin{array}{ll}0.5 & -1.5\end{array}\right]^{T}$ under disturbance, given by:

$$
\omega=\left[\begin{array}{lll}
0.1 \sin \left(x_{1}\right) & 0.12 \sin \left(x_{2}\right) & 0.15 \sin \left(x_{1}\right)
\end{array}\right]^{T} .
$$

From the simulation, we know that the effect of state trajectory tracking is satisfactory. 


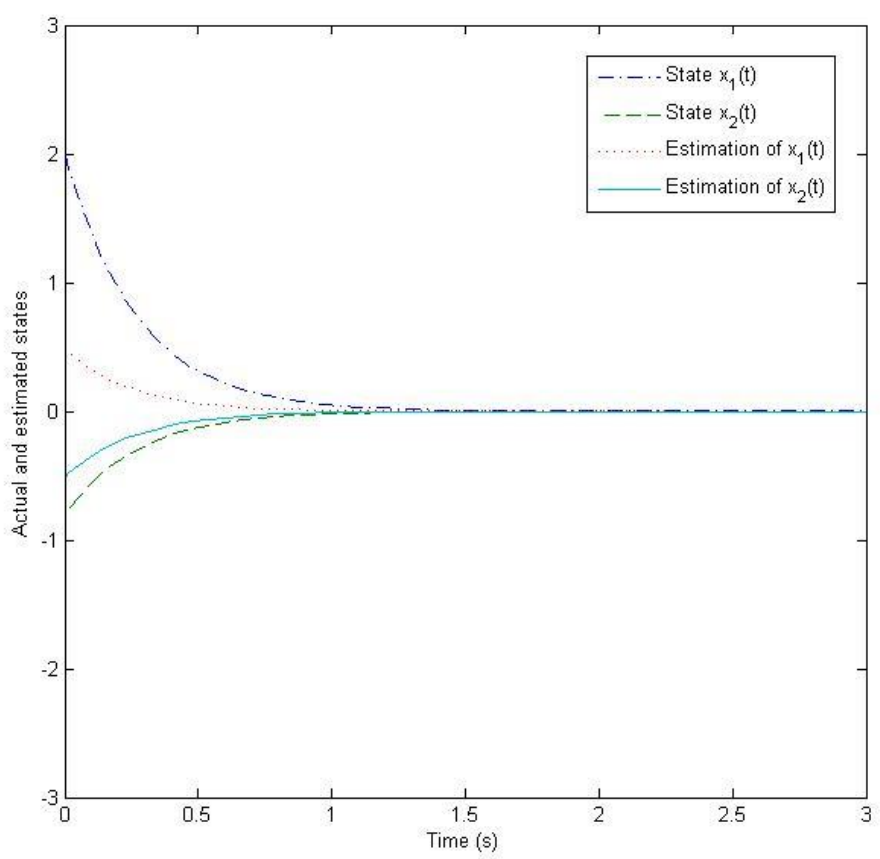

Figure 1: Convergence of the observer state $\hat{x}$ to the plant state $x$.

\section{Conclusion}

In this paper, the problem of observer design of descriptor time delay system is studied in terms of the onesided Lipschitz condition and quadratic internal boundary condition. Through the construction of the LyapunovKrasovskii functional, the BMI-based sufficient conditions are obtained to ensure asymptotic convergence of the state estimation error to origin and to accomplish robustness against $L_{2}$ norm bounded disturbances. Based on the conditions, the observer gain matrix is obtained by substituting appropriate variables. Finally, a numerical example and simulation results are given. To further the findings, future studies can take parameter uncertainties and state and output delays into consideration.

\section{Acknowledgement}

This work is supported by the National Nature Science Foundation of China under Grant 61703307.

\section{References}

[1] R. Sakthivel, S. Santra, K. Mathiyalagan, Admissibility analysis and control synthesis for descriptor systems with random abrupt changes, Appl. Math. Comput, 2013, 219: 9717 - 9730.

[2] Z.-G. Wu, J. H. Park, H. Su, J. Chu, Reliable passive control for singular systems with time-varying delays, J. Process Control, 2013, 23: 1217 - 1228.

[3] P. Liu, W.-T. Yang, C.-E. Yang, Robust observer-based output feedback control for fuzzy descriptor systems, Expert Syst. Appl, 2013, 40: 4503 - 4510.

[4] F. Zhao, Q. Zhang, X. Yan, $H_{\infty}$ filtering for stochastic singular fuzzy systems with time-varying delay, Nonlinear Dyn, 2015, 79(1): 215 - 228.

[5] M. Abbaszadeh, H. Marquez, Nonlinear observer design for one-sided Lipschitz systems, in: Proceedings of the American Control Conference Baltimore, USA, 2010, pp. 5284 - 5289.

[6] G. Hu, Observers for one-sided Lipschitz nonlinear systems, IMAJ. Math. Control Inf, 2006, 23(4): 395 401.

[7] W. Liu, Y. Dong, S. Zuo, Design of observer for one-sided Lipschitz nonlinear systems with interval timevarying delay, Wseas Transactions on Systems and Control, 2015, 10: 385-395.

[8] W. Zhang, H. Su, H. Wang, Z. Han, Full-order and reduced-order observers for one-sided Lipschitz nonlinear systems using Riccati equations, Commun. NonlinearSci. Numer. Simul., 2012, 17: 4968 - 4977. 
[9] M. Benallouch, M. Boutayeb, M. Zasadzinski, Observer design for one-sided Lipschitz discrete-time systems, Syst. Control Lett., 2012, 61: $879-886$.

[10] W. Zhang, H. Su, S. C. Su, D. Z. Wang, Nonlinear $H_{\infty}$ observer design for one-sided Lipschitz systems, Neurocomputing, 2014, 145: 505 - 511.

[11] G. L. Wang, H. Y. Bo, Q. L. Zhang, $H_{\infty}$ filtering for time-delayed singular Markovian jump systems with time-varying switching: a quantizes method. Signal Process, 2015, 109:14 - 24.

[12] Y. Dong, J. Liu, S. Mei, Observer design for a class of nonlinear discrete-time systems with time-delay, Kybernetika, 2013, 49(2): 2342-359.

[13] A. Zemouche, M. Boutayeb, Observer synthesis method for Lipschitz non-linear discrete time systems with time delay: an LMI approach. Appl. Math. Comput., 2011, 218(2): 419-429.

[14] S. Yia, A. G. Ulsoya, P. W. Nelsonb, Design of observer-based feedback control for time delay systems with application to automotive powertrain control, Journal of the Franklin Institute, 2010, 347(1): 358-376.

[15] Y. Dong, H. Wang, Y. Wang, Design of observers for nonlinear systems with $H_{\infty}$ performance analysis, Mathematical Methods in the Applied Sciences, 2014, 37(5): 718-725.

[16] R. A. Horn, C. R. Johnson, Matrix analysis, Cambridge University Press, 1985. 